\title{
Capital social en la perspectiva de la teoría de recursos y capacidades: un análisis bibliométrico
}

\author{
Volumen XIX No (1). Enero-Junio 2019. Pág. 63-77
}

ISSN: 0121-1048 IMPRESO ISSN: 2422-3220 EN LÍNEA

\author{
López-Inda, Karina \\ Azucena* \\ Universidad Autónoma de \\ Sinaloa, Facultad de \\ Contaduría y \\ Administración, Ciudad \\ Universitaria Módulo 4, \\ Col. Villa Universidad, \\ 80010 Culiacán. Sinaloa \\ México. \\ Universidad Autónoma de \\ Occidente, Unidad \\ Culiacán, Lola Beltrán $S / n$, \\ Col. 4 de Marzo, 80120, \\ Culiacán, Sinaloa, México. \\ klopezinda@yahoo.com.mx \\ *Autor corresponsal
}

\author{
Alvarado Altamirano, \\ Sergio \\ Universidad Autónoma de \\ Occidente, Unidad \\ Culiacán, Lola Beltrán $S / n$, \\ Col. 4 de Marzo, 80120, \\ Culiacán, Sinaloa, México. \\ sergalva60@gmail.com
}

\section{Fong Reynoso, Carlos}

Universidad de

Guadalajara, Centro

Universitario de Ciencias

$$
\text { Económico }
$$

Administrativas,

Periférico Norte $N^{\circ} 799$,

Núcleo Universitario Los

Belenes, 45100. Zapopan,

Jalisco, México.

smart.fong.mx@gmail.co

$m$

\section{González Álvarez, Eleazar}

Universidad Autónoma

de Sinaloa, Facultad de

Contaduría y

Administración, Ciudad

Universitaria Módulo 4,

Col. Villa Universidad,

80010 Culiacán. Sinaloa,

México.

eleazargonzalez.fca@gma

il.com

\section{Resumen}

En este estudio se realiza una aproximación a la perspectiva integrada del capital social bajo la Teoría de recursos y capacidades. Para ello se realizó un análisis bibliométrico de citación, palabras clave y autoría de la literatura relevante contenida en la base de datos Scopus. El análisis se hizo con el apoyo de las herramientas Excel y VOSviewer. Así mismo, se aplicó una revisión de contenido a los 46 documentos que arrojó la búsqueda. Los resultados confirman que el capital social es tratado en la literatura especializada como un recurso que contribuye a mejorar el desempeño de la empresa y la formación de ventaja competitiva, además de estar estrechamente vinculado al uso del capital humano y al desarrollo de capacidades como la innovación, el aprendizaje, la adaptación, así como a la gestión del conocimiento .

Palabras clave: Teoría de recursos y capacidades (TRC), capital social, ventaja competitiva, innovación

Códigos JEL: M10, M53, O12, O31.

\footnotetext{
• Citar: López-Inda, Karina Azucena, Alvarado Altamirano, Sergio, Fong Reinoso, Carlos y González Álvarez, Eleazar (2019). Capital social en la perspectiva de la teoría de recursos y capacidades: un análisis bibliométrico. Inquietud Empresarial, XIX (1), 63-77.
} 


\title{
Social capital in the perspective of the theory of resources and capabilities: a bibliometric analysis
}

\begin{abstract}
In this study, a first approach is made to the integrated perspective of social capital under the Theory of Resources and Capabilities. For this purpose, the Scopus database was selected, from which the necessary information was extracted to perform a bibliometric analysis of citation, keywords and authorship with the support of the Excel and VOSviewer tools. Likewise, a content review was applied to the 46 documents that the search showed. The results confirm that social capital is seen as a firm resource that contributes to improving performance and the formation of sustainable competitive advantages, as well as being closely linked to the use of human capital and the development of skills such as innovation, learning, adaptation, as well as knowledge management.
\end{abstract}

Keywords: Resources and capabilities theory (RBT), social capital, competitive advantage, innovation

JEL Codes: M10, M53, O12, O31.

\section{INTRODUCCIÓN}

En los años recientes, los estudios sobre el desarrollo económico regional, innovación y competitividad han prestado una creciente atención al rol que desempeña el capital social en dichos procesos. El capital social se define como el conjunto de recursos incorporados y disponibles por medio de la red de relaciones sociales que posee un individuo o grupo, así como otros factores que intervienen para explicar el rendimiento económico exitoso de una empresa, una región o un país. Dentro de dichos estudios, una de las teorías que se considera como mejor explicación del éxito de la empresa es la Teoría de Recursos y Capacidades (en adelante TRC) (Barney 1991; Barney, Wright y Ketchen 2011). La creciente aceptación y utilización de la TRC como explicación del desempeño de la empresa se encuentra en la observación empírica acumulada en la literatura internacional acerca de que los factores internos de la empresa, y no tanto los factores externos, son los explican en mayor medida los resultados logrados por las empresas (Fong, Flores y Cardoza, 2017).

De acuerdo con la TRC, la ventaja competitiva de la empresa depende primordialmente de la naturaleza de sus recursos y capacidades, así como de la forma en que estos se usan y gestionan en el marco de la estrategia de la empresa (Peteraf, 1993; Habbershon y Williams, 1999; Becattini, 2006; Helfat y Martin, 2015; Schenkel y Teigland, 2017). Por consiguiente, y con el objetivo de realizar un análisis que permita explorar la perspectiva más reciente del capital social bajo la TRC, se plantea a continuación un ejercicio bibliométrico que permite identificar el estado actual de la producción científica en este campo.

No está de más describir que el análisis bibliométrico consiste en una técnica que permite evaluar, por medio de indicadores, el impacto de una publicación individual o de una revista; la influencia que esta ha tenido en sus receptores; la relevancia científica y la articulación disciplinaria; así como identificar fortalezas y oportunidades de investigación; 
identificar tendencias, modas, eslabonamientos y callejones sin salida, clústeres de conocimientos, de comunidades científicas y de redes; clasificar autores según su productividad; además de identificar instituciones y países en los que se están generando los artículos científicos; obras más influyentes, entre otras posibilidades (Arbeláez y Onrubia, 2014). Como se puede ver, sus usos son muy variados, por lo que se hace hincapié que para el presente análisis se emplearon algunos indicadores cuantitativos como número de citas por documento, número de publicaciones por año y por área temática, así como de contenido tales como palabras clave dentro del título y/o resúmenes, dado que el propósito fue revisar los textos de acuerdo con el objetivo de investigación.

Asimismo, el análisis bibliométrico ha sido ampliamente utilizado en diversas disciplinas como salud, medicina, negocios, sustentabilidad, sistemas e informática, y su validez queda expuesta en trabajos como el de Merigó, Gil-Lafuente y Yager (2015), Pineda (2015), Portugal et al (2016), Blanco-Mesa, Gil-Lafuente y Merigó (2017), Fong et al (2017), van Numen (2017), Garrigos-Simon, Botella-Carrubi y González-Cruz (2018), Wang y Zhao (2018) quienes destacan cómo este tipo de análisis permite identificar posturas y tendencias en el conocimiento por medio de la revisión bibliográfica cuantitativa y cualitativa.

De esta manera, la idea central que guía este estudio es que el capital social es un recurso valioso de las empresas que les posibilita la generación de un mayor desempeño y crecimiento (Nahapiet y Ghoshal, 1998; Lee, Lee y Pennings, 2001; Anand, Glick y Manz, 2002; Becattini, 2006; Helfat et al, 2007; Abili, 2011). Estos mismos investigadores sugieren que el capital social proporciona un medio para aprovechar el conocimiento tácito de los demás, tener disponibilidad y acceso a información, fomentar la confianza y la solidaridad, y hacer uso de la influencia y el poder de los miembros cuando se asocian para trabajar de forma colaborativa.

En síntesis, se pretende indagar si en los últimos años esta idea ha prevalecido o bien, cuál es la postura que sostienen las nuevas investigaciones. Y no solamente con respecto a que el capital social provee recursos, como se ha señalado, sino también buscando eco en la literatura reciente sobre si el uso de esos recursos ayuda a generar capacidades específicas no imitables ni transferibles que garanticen una ventaja competitiva sostenible para la empresa.

\subsection{MARCO TEÓRICO}

\subsection{Competitividad y ventaja competitiva}

Lograr el desarrollo económico de una región, que garantice un elevado nivel de bienestar social y económico a su población, depende de que las empresas instaladas en su territorio cuenten con la capacidad suficiente para lograr altos niveles de productividad y competitividad (Porter, 1990; Florida, 2010). Aunque en un principio se consideró que un factor determinante del éxito de las empresas estaba dado por los atributos de su líder, bastaron algunas décadas para que ellas mismas comprobaran que esto no era suficiente, necesitaban todo un equipo de directivos altamente competente, además de considerar otros factores clave como el posicionamiento de mercado, la calidad de los productos, el capital humano, la innovación y la tecnología (Mintzberg, Lampel y Ahlstrand, 1999). Esto con la finalidad de 
desarrollar nuevas y mejores estrategias que les permitieran hacer frente a un entorno cada vez más complejo y cambiante.

\subsubsection{Desarrollo de estrategias competitivas}

Fue entonces que la atención se centró en el binomio estrategia-entorno, por esta razón, el modelo estructural ampliamente referenciado en la literatura y utilizado en el ambiente empresarial y económico, conocido como Las cinco fuerzas de Porter (1990) es un claro ejemplo de ello. En general, los desarrollos teóricos de Porter definen a una empresa competitiva cuando esta consigue de forma sostenida en el tiempo, una rentabilidad superior a la media del sector o industria en la que compite, desarrollando para ello estrategias específicas.

El problema con las estrategias es que son hasta cierto punto imitables, por lo que efectivamente pueden proporcionar ventajas, pero sólo a un corto plazo. Así, a fines de los ochenta y principios de los noventa, al percatarse que no todas las empresas lograban desarrollar y mantener una ventaja competitiva, los investigadores comenzaron a focalizar sus esfuerzos hacia una dimensión intraorganizacional, buscando aquél elemento diferenciador, que para Penrose (1959), Nelson y Winter (1982), Wernerfelt (1984), Rumelt (1984), Grant (1991), Mahoney y Pandian (1992), Peteraf (1993), Habbershon y Williams (1999), ha sido la disponibilidad de recursos heterogéneos y el uso particular que se hacía de ellos conformando las llamadas capacidades internas de la empresa, debido a su contribución a la ventaja competitiva. Después de esto, el problema para los directivos ha sido identificar, desarrollar, proteger y desplegar los recursos $y$ capacidades necesarios en aquellos mercados donde se pueda alcanzar ventajas competitivas sostenibles (Ventura, 2008). Adicionalmente, resulta útil, en grado superlativo, el hecho de adoptar un enfoque integrado de múltiples teorías para explicar el crecimiento moderno de la empresa a partir de la teoría de Penrose y la TRC (Burvill, Jones-Evans y Rowlands, 2018).

\subsection{Teoría de recursos y capacidades}

La integración de teorías señalada en el apartado anterior propició que la atención se concentrara en el estudio de las variables internas de la empresa que constituían la base de su estrategia y por consiguiente de su rentabilidad y competitividad, sentando las bases para el surgimiento de la llamada Teoría de Recursos y Capacidades (TRC), la cual gira en torno a dos ideas principales: 1) las empresas son heterogéneas por naturaleza y, por ende, 2) sólo pueden desarrollar una ventaja competitiva sostenible por la accesibilidad y combinación de recursos únicos no disponibles para sus competidores (Rumelt, 1984; Wernerfelt, 1984; Barney, 1991). A su vez, para que un recurso contribuya a la generación de la ventaja competitiva debe poseer cuatro atributos: 1) debe ser valioso, en el sentido de que permite aprovechar las oportunidades o neutralizar las amenazas del entorno de la empresa, 2) debe ser raro entre los competidores actuales y potenciales de una empresa, 3) debe ser imperfectamente imitable y 4) no debe tener sustitutos estratégicamente equivalentes (Barney, 1991). En general, estos planteamientos se mantienen vigentes hasta el día de hoy, sin embargo, cabe señalar que para el caso de las economías emergentes es más recomendable emplear el enfoque de capacidades dinámicas, concepto que será desarrollado en párrafos posteriores (Helfat y 
Peteraf, 2003; Peteraf, Di Stefano y Verona, 2013).

\subsubsection{Perspectiva del capital social bajo la teoría de recursos y capacidades}

Ahora bien, partiendo de la idea que prevalece en los autores clásicos como Bourdieu (1980), Coleman (1988), Granovetter (1992), Putnam (1993), Nahapiet y Ghoshal (1998), el capital social es un conjunto de recursos específicos que posee la empresa, ya sean materiales, financieros, conocimiento, información, contactos e influencias, entre otros, que se pueden movilizar a través de las diferentes relaciones que se establecen entre los miembros, las cuales facilitan y promueven la cooperación generando beneficios para los participantes, dentro del marco de un conjunto de normas y otros elementos como confianza, valores y objetivos compartidos, de ahí que el propósito de esta investigación es tomar como base la TRC para poner de manifiesto la naturaleza de dicho capital y qué circunstancias posibilitan la creación de capacidades específicas no imitables ni transferibles que garanticen ventajas competitivas sostenibles.

Como se puede apreciar, el capital social se anida en las relaciones interpersonales, idea que también es apoyada por investigaciones recientes que ponen de manifiesto la importancia de construir redes de colaboración entre individuos de una organización y de estos con otras organizaciones, de manera que se facilite $y$ promueva el acceso a recursos de todo tipo, generando con ello beneficios como confianza, satisfacción, liderazgo, compromiso, innovación y conocimiento, lo que a su vez incida en un desempeño superior de los participantes, en el marco de normas que moderen dichas relaciones sociales (Lees y Nuthall, 2015; Overall, 2015; Whipple, Wiedmer, y Boyer, 2015; Tomlinson y Fai, 2016; Jalali, Thurasamy y Jaafar, 2017; Muldoon, Bauman y Lucy, 2018).

Con esto los autores, tanto clásicos como contemporáneos, hacen referencia a que es más probable que un individuo $u$ organización logre sus objetivos de manera puntual al establecer lazos con otros individuos o grupos, a la vez que intercambia y comparte sus recursos con ellos.

\subsubsection{Capital social como recurso intangible}

Siguiendo a Penrose (1959), Andrews (1971) y Barney (1991), los recursos son los activos financieros, materiales, tecnológicos, humanos $\mathrm{y}$ organizativos disponibles $\mathrm{y}$ controlables por la empresa. Dicho en términos más generales, para Wernerfelt (1984) un recurso es todo aquello susceptible de ser reconocido como una fortaleza de la empresa e identificable como un activo, ya sea tangible o intangible. Los recursos tangibles hacen referencia a todo aquello que pueda ser registrado como un bien que es propiedad de la empresa, mientras que los intangibles son aquellos que aunque no se puedan percibir directamente, están presentes y forman parte de los procesos productivos, organizativos y sociales de la misma.

Con respecto a estos últimos, Bueno y Morcillo (1992) afirman que los recursos intangibles son los que configuran hoy la base de la competitividad empresarial. Adicionalmente, Rugman y Verbeke (2002) sostienen que, dentro de este conjunto de recursos, los ligados al capital humano y la gestión de los mismos, son los más difíciles de imitar pues aluden al conocimiento y las relaciones sociales, dos elementos intrínsecamente complejos (Overall, 2015; 
Whipple et al, 2015; Pee y Kankanhalli, 2016; Muldoon et al, 2018).

\subsubsection{Capital social y su relación con las capacidades de la empresa}

Como ya se estableció anteriormente, el capital social es un recurso intangible que nace de las relaciones interpersonales, sin embargo, aún no se tiene claro cuáles son los factores que motivan dichas relaciones, es decir, qué es lo que hace que los individuos o grupos establezcan lazos de amistad, confianza, lealtad, solidaridad y compartan sus propios recursos. No obstante es un hecho que para obtener y acrecentar el capital social se requiere que las personas desplieguen algunas capacidades dadas por su capital humano como conocimiento, experiencia y habilidades (Castanias y Helfat, 2001; Ostrom y Ahn, 2003) y, aunque la literatura no especifica de qué tipo, deben servir para construir relaciones sociales útiles $\mathrm{y}$ duraderas, por lo que de acuerdo con la experiencia, se infiere que estas habilidades son las comunicativas, de relaciones personales, toma de decisiones y empatía.

De la misma forma que se requieren ciertas capacidades para adquirir y mantener los recursos que conforman el capital social, así también, se precisa de otras capacidades para explotar y maximizar dichos recursos (Helfat y Martin, 2015; Singh y Rao, 2016; Pee y Kankanhalli, 2016). Esto significa que no es suficiente poseer los recursos, sino que estos deben ser utilizados de manera única y diferenciada, para lo cual la empresa debe ser capaz de desarrollar capacidades distintivas que le posibiliten la creación e implantación de estrategias dominantes y efectivas en un entorno en constante cambio.

En este sentido, vale la pena aludir al enfoque denominado "capacidades dinámicas" propuesto por Teece, Pisano y Shuen (1997) y que viene a complementar a la TRC de Barney (1991). Esto considerando que el contexto cambia rápidamente y las empresas tienen que actuar en consecuencia, construyendo capacidades dinámicas sobre la base de sus recursos disponibles, competencias, procesos definidos y estrategias implementadas en el pasado. Por lo que, a partir de estos elementos, las capacidades dinámicas se definen como aquellas habilidades para crear, combinar o modificar la base de recursos que posee la empresa, de tal forma que le permitan generar bienes y servicios capaces de participar mercados altamente competitivos (Teece y Pisano, 1994; Teece et al, 1997; Zollo y Winter, 2002; Helfat et al, 2007).

Se puede inferir entonces que, una vez adquirido el capital social, los directivos y sus colaboradores requieren poner en práctica habilidades, conocimiento y experiencia para hacer uso de este capital, de tal forma que contribuya a potenciar otras capacidades que faciliten la movilización de recursos que se orienten a buscar nuevas oportunidades de negocio (Helfat y Martin, 2015; Singh y Rao, 2016; Schenkel y Teigland, 2017; Huy y Zott, 2019). Estas capacidades pueden ser la capacidad de innovación, capacidad de absorción, capacidad de aprendizaje, capacidad de adaptación (estas cuatro establecidas como capacidades dinámicas por Garzón 2015), la inteligencia competitiva y la gestión del conocimiento (Ventura, 2008). Todas ellas, habilidades necesarias para que la empresa genere algún tipo de ventaja competitiva que perdure en el tiempo debido a su dificultad de ser imitada, adquirida o sustituida. 


\section{METODOLOGÍA}

Los datos para realizar este estudio se obtuvieron de la base de datos Scopus, por ser una de las mayores bases de datos disponibles en la actualidad, que alberga tanto revistas científicas, como libros y actas de congresos que proveen información sobre el quehacer de la investigación mundial en los campos de la ciencia, la tecnología, la medicina, las ciencias sociales, así como las artes y las humanidades (tomado de su página web). Además, por ser una base cuyos datos pueden ser extraídos en formato Excel extensión .CSV el cual es compatible con el software VOSviewer utilizado para generar los mapas que se presentan en este documento. La búsqueda se realizó durante el periodo del 10 al 23 de septiembre y del 12 al 22 de octubre de 2018, concluyendo con la descarga final de los datos el 24 de octubre.

Dicha búsqueda se realizó en idioma inglés y se aplicaron una serie de combinaciones de palabras clave que permitieran definir el perfil de los artículos a elegir. El resultado de ello fue la selección de las palabras "social capital" seguida de (AND) "resource-based view", "resource-based theory", RBV (Fong, 2017) para estas tres últimas se utilizó el operador lógico OR, y se condicionó a la coincidencia en título, resumen y palabras clave de los documentos publicados. La primera búsqueda arrojó un total de 123 documentos en todos los años de publicación de Scopus, sin distinción de autores y en todas sus áreas temáticas. Este número se vio reducido al filtrar la búsqueda a las áreas de interés para este artículo, las cuales, además, representan el mayor número de documentos publicados que son las de negocios, administración y contabilidad, economía y finanzas, y ciencias sociales, obteniendo la cantidad de 112 resultados, de estos, filtrando de acuerdo a los últimos cinco años (del 2014 al 2018) quedaron 46, los cuales constituyen la base de documentos que se revisaron para realizar el presente estudio y aunque el $80 \%$ corresponden a artículos científicos, se tomaron en cuenta todos los tipos de documento por considerar que ayudarían a reforzar la base.

Los resultados de la búsqueda se exportaron a archivos Excel por ser un programa de uso generalizado que permite además del almacenamiento, la organización y manipulación de datos, para posteriormente poder representarlos en tablas y gráficos, en este caso de acuerdo al propósito de este estudio. También se realizó la exportación de archivos Excel con extensión .CSV, los cuales se generaron para ser utilizados en el software VOSviewer para la creación de los mapas de citación, palabras clave y autoría.

Cabe señalar que, por el hecho de haber realizado este estudio con la ayuda de una base de datos, puede existir la posibilidad de que la misma no incluya algunos documentos debido a los periodos de tiempo de publicación o a las áreas temáticas que maneja y a sus procesos de actualización. Asimismo, se tomaron en cuenta solamente los documentos que resultaron de los criterios de búsqueda aplicados y en las fechas en que esta fue realizada, por lo que no se debe considerar que este trabajo representa el conocimiento total que existe en este campo de investigación. Incluso, existe la posibilidad de que al modificar los criterios de búsqueda los resultados que se arrojen sean diferentes a los aquí mostrados. 


\section{RESULTADOS}

Como se mencionó en el apartado anterior, la base de datos con la cual se trabajó para este estudio fue extraída de Scopus y está conformada por 46 documentos, de los cuales 37 corresponden a artículos, cinco a capítulos de libro, dos artículos en prensa, un paper de conferencia y uno de revisión, todos ellos publicados durante el periodo 2014-2018.
Aunque la investigación sobre capital social, lo mismo que la de TRC, no es reciente, la revisión arroja que existe poca producción en este periodo, siendo el año 2016 el que tiene mayor registro de publicaciones asociadas con dicha temática (ver gráfica 1). Lo que permite observar que el capital social, analizado desde la perspectiva de esta teoría, aún es parte de una temática poco explorada, no obstante, se espera que en unos años más la tendencia a investigar sobre ella siga en aumento como se aprecia en la gráfica.

GRÁFICA 1. NÚMERO DE PUBLICACIONES POR AÑO

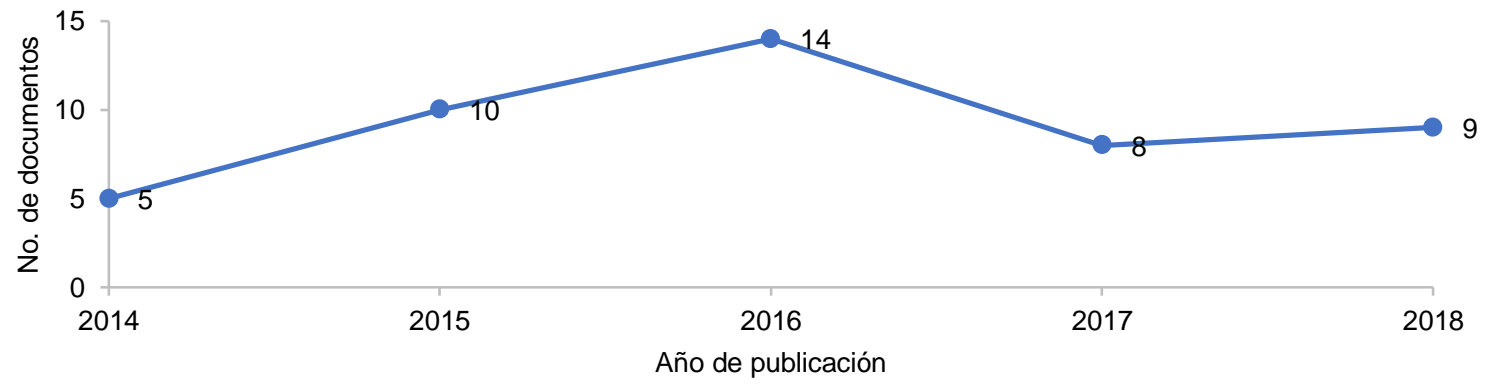

Fuente: (Base de datos Scopus)

También se identificó que las áreas en las que mayormente se han realizado estudios relacionados con el capital social y la TRC continúan siendo las administrativas $\mathrm{y}$ sociales, como señala la literatura. Con relación a la cantidad de documentos publicados sobresalen las áreas de Negocios, Administración y Contabilidad con un 42.5\%, Economía y Finanzas con 19.5\%, y Ciencias Sociales con $13.8 \%$ (ver gráfico 2).

GRAFICA 2. NÚMERO DE PUBLICACIONES POR ÁREA.

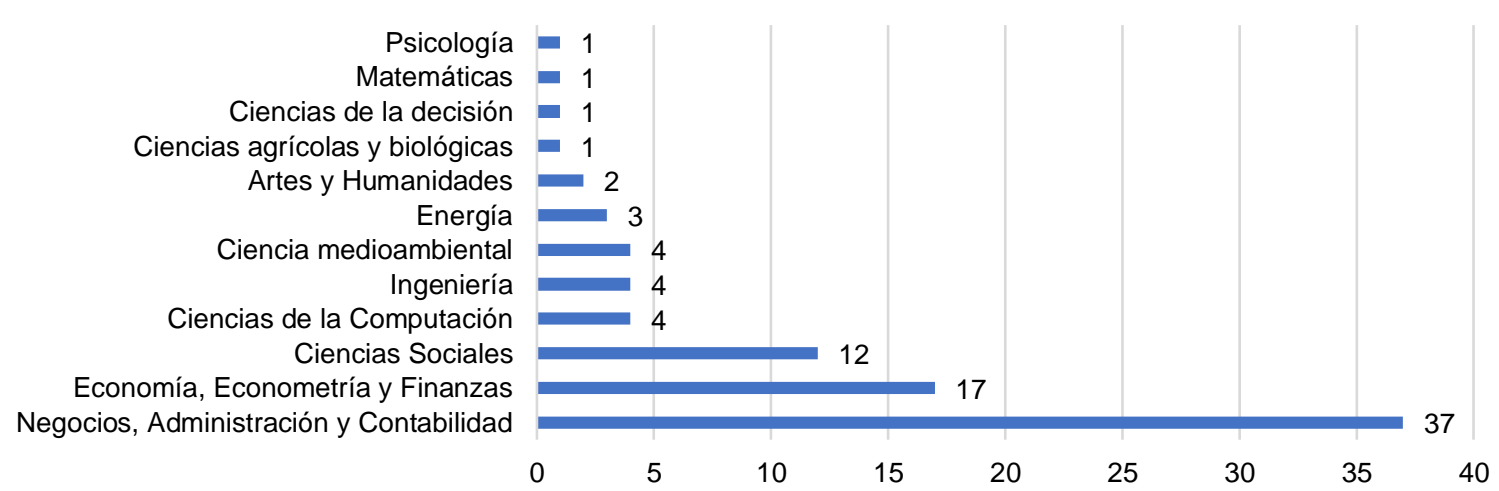

Source: own development 
Como resultado del tratamiento de la información sobre citación, palabras clave y autoría por medio del software VOSviewer, se obtuvo que las palabras con mayor número de coincidencias en los documentos analizados son, además de capital social (social capital) y la TRC (resourced-based view or resource-based theory, la de capital humano (human capital), que como ya se mencionó es posible considerarlo como una capacidad que impulsa y promueve la formación de capital social, así como la variable innovación (innovation), la cual se considera como una capacidad dinámica susceptible de ser generada a partir del uso de los recursos que proporciona el capital social (ver Figura 1).

FigURA 1. PALABRAS CLAVE IDENTIFICADAS EN LOS DOCUMENTOS.

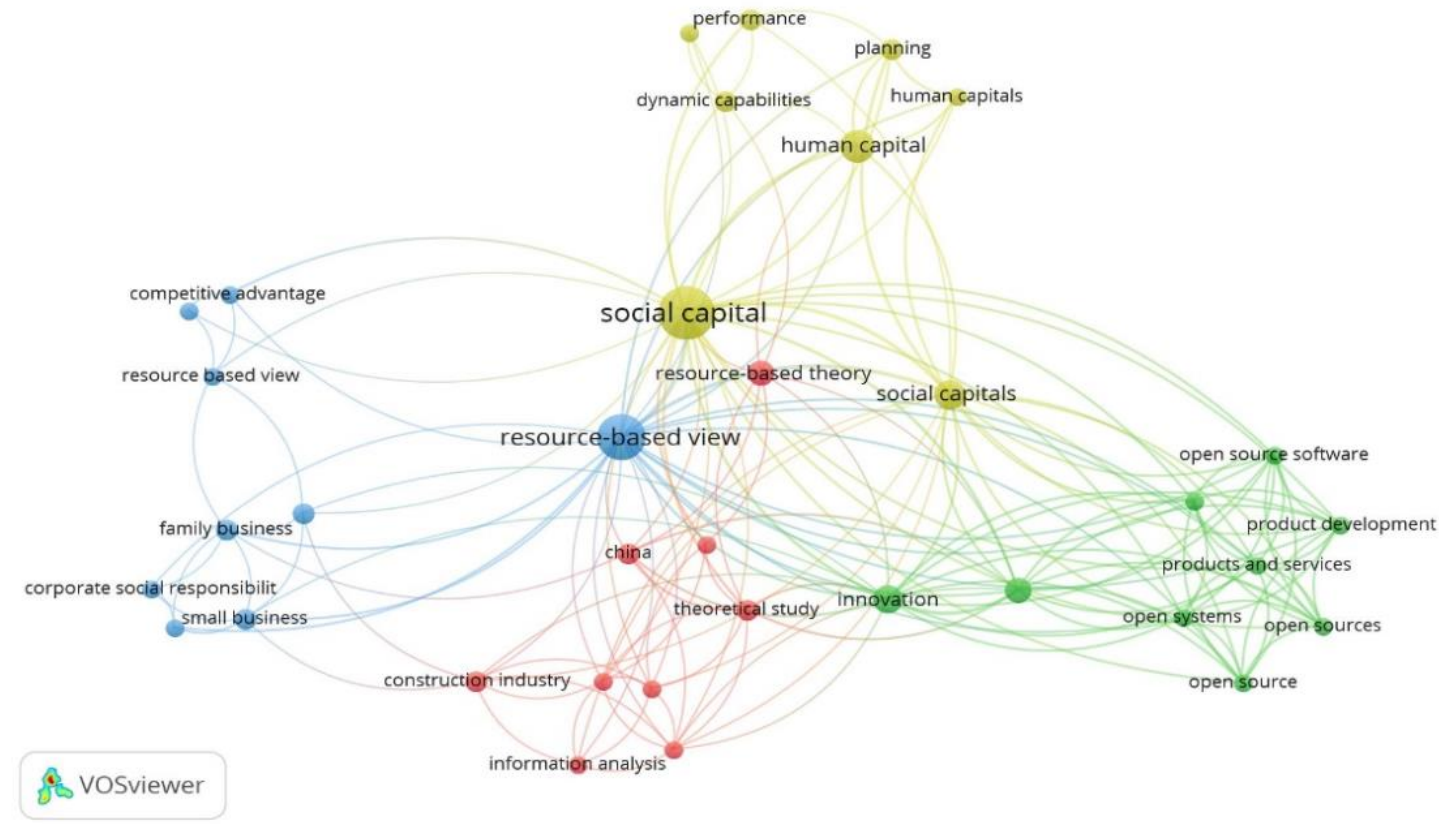

Fuente: (Base de datos Scopus) y VOSviewer

Se puede observar claramente cómo estos constructos a su vez conforman clústeres o grupos de palabras relacionadas con ellos, resaltando que el capital social y el capital humano tienen una fuerte conexión, ya que junto con las capacidades dinámicas promueven un mayor desempeño de la organización.

En este análisis también se identificó que el artículo más referenciado es Dynamic Managerial Capabilities: Review and Assessment of Managerial Impact on
Strategic Change de Helfat y Martin (2015) con 72 citas, seguido del artículo Status and reputation: Synonyms or separate concepts? de Sorenson (2014) con 22, así como el de Miller (2015) y Whipple et al (2015) con 20 (ver Tabla 1). El artículo de Helfat y Martin (2015) centra su atención sobre las capacidades gerenciales dinámicas y las clasifica en tres dimensiones: cognición gerencial, capital social gerencial y capital humano gerencial. Asimismo, presenta un análisis de cómo estas capacidades influyen 
en la calidad de las decisiones de los gerentes y estas a su vez en el cambio estratégico y el desempeño organizacional, por lo que los resultados varían de una empresa a otra debido a que cada gerente actúa en función de dichas capacidades. Mientras que el de Sorenson (2014) analiza las diferencias y similitudes de dos factores: status $y$ reputación de la empresa, los cuales a menudo son considerados como sinónimos, pero que para él son diferentes $y$, por lo tanto, así deberían ser tratados, además, algo que tiene en común es que ambos pueden ser considerados como recursos intangibles que forman parte del capital social de cualquier organización y que le pueden representar en un momento dado una ventaja competitiva.

TABLE 1. DOCUMENTOS CON MAYOR NÚMERO DE CITAS

\begin{tabular}{|c|c|c|c|c|}
\hline No. & Título & Autor (es) & Año & $\begin{array}{ll}\begin{array}{l}\text { No. de } \\
\text { citas }\end{array} \\
\end{array}$ \\
\hline 1 & $\begin{array}{l}\text { Dynamic Managerial Capabilities: Review and Assessment of } \\
\text { Managerial Impact on Strategic Change }\end{array}$ & Helfat C.E., Martin J.A. & 2015 & 72 \\
\hline 2 & Status and reputation: Synonyms or separate concepts? & Sorenson O. & 2014 & 22 \\
\hline 3 & $\begin{array}{l}\text { A Dyadic investigation of collaborative competence, social } \\
\text { capital, and performance in buyer-supplier relationships }\end{array}$ & $\begin{array}{l}\text { Whipple J.M., Wiedmer } \\
\text { R., Boyer K.K. }\end{array}$ & 2015 & 20 \\
\hline 4 & $\begin{array}{l}\text { When is human capital a valuable resource? the performance } \\
\text { effects of Ivy league selection among celebrated CEOs }\end{array}$ & $\begin{array}{l}\text { Miller D., Xu X., Mehrotra } \\
\text { V. }\end{array}$ & 2015 & 20 \\
\hline 5 & $\begin{array}{l}\text { Boards and Sustainability: The Contingent Influence of } \\
\text { Director Interlocks on Corporate Environmental Performance }\end{array}$ & $\begin{array}{l}\text { Ortiz-de-Mandojana N., } \\
\text { Aragon-Correa J.A. }\end{array}$ & 2015 & 14 \\
\hline 6 & $\begin{array}{l}\text { Suppliers' communication capability and external green } \\
\text { integration for green and financial performance in Korean } \\
\text { construction industry }\end{array}$ & $\begin{array}{l}\text { Woo C., Kim M.G., Chung } \\
\text { Y., Rho J.J. }\end{array}$ & 2016 & 13 \\
\hline 7 & $\begin{array}{l}\text { Impacts of leadership on project-based organizational } \\
\text { innovation performance: The mediator of knowledge sharing } \\
\text { and moderator of social capital }\end{array}$ & Zheng J., Wu G., Xie H. & 2017 & 10 \\
\hline 8 & $\begin{array}{l}\text { Interactions among factors influencing knowledge } \\
\text { management in public-sector organizations: A resource-based } \\
\text { view }\end{array}$ & Pee L.G., Kankanhalli A. & 2016 & 10 \\
\hline 9 & $\begin{array}{l}\text { Organizational effectiveness, people and performance: new } \\
\text { challenges, new research agendas }\end{array}$ & Sparrow P., Cooper C. & 2014 & 9 \\
\hline 10 & $\begin{array}{l}\text { How network-based incubation helps start-up performance: a } \\
\text { systematic review against the background of management } \\
\text { theories }\end{array}$ & $\begin{array}{llr}\text { Eveleens } & \text { C.P., } & \text { van } \\
\text { Rijnsoever } & \text { F.J., Niesten } \\
\text { E.M.M.I. } & & \end{array}$ & 2017 & 7 \\
\hline
\end{tabular}

Fuente: (Base de datos Scopus).

El resultado anterior se complementa con el mapa topológico de la red de autores presentado en la Figura 2, en el cual se destaca la productividad en número de citas de los autores Helfat (2015), Sorenson (2014), Miller (2015), Whipple et al (2015) y Ortiz de Mandojana (2015).

En este caso vale la pena remarcar que los autores no presentaron ninguna conexión, lo que significa que no existe un trabajo colaborativo de trascendencia en este ámbito. Además de que todos ellos presentan solamente una publicación, es decir, la producción de artículos que relaciones al capital social con la TRC aún es limitada.

Por otra parte, se realizó una revisión detallada de los documentos para explorar la perspectiva más reciente del capital social combinado con la TRC, encontrando que, en la mayoría de los artículos, se le da el tratamiento de recurso, pero no un recurso ordinario que cualquiera puede poseer, sino de manera particular aquél que poseen los fundadores, directores, gerentes y que incide directamente en el desempeño de sus empresas (Casanueva, Gallego y Revilla, 
2015; Helfat y Martin, 2015; Huynh y Patton, 2015; Miller, Xu y Mehrotra, 2015; Krause,
Semadeni y Withers, 2016; Muttakin, Khan y Mihret, 2018; Wang, Huang y Liu, 2018).

FIGURA 2. PALABRAS CLAVE IDENTIFICADAS EN LOS DOCUMENTOS.

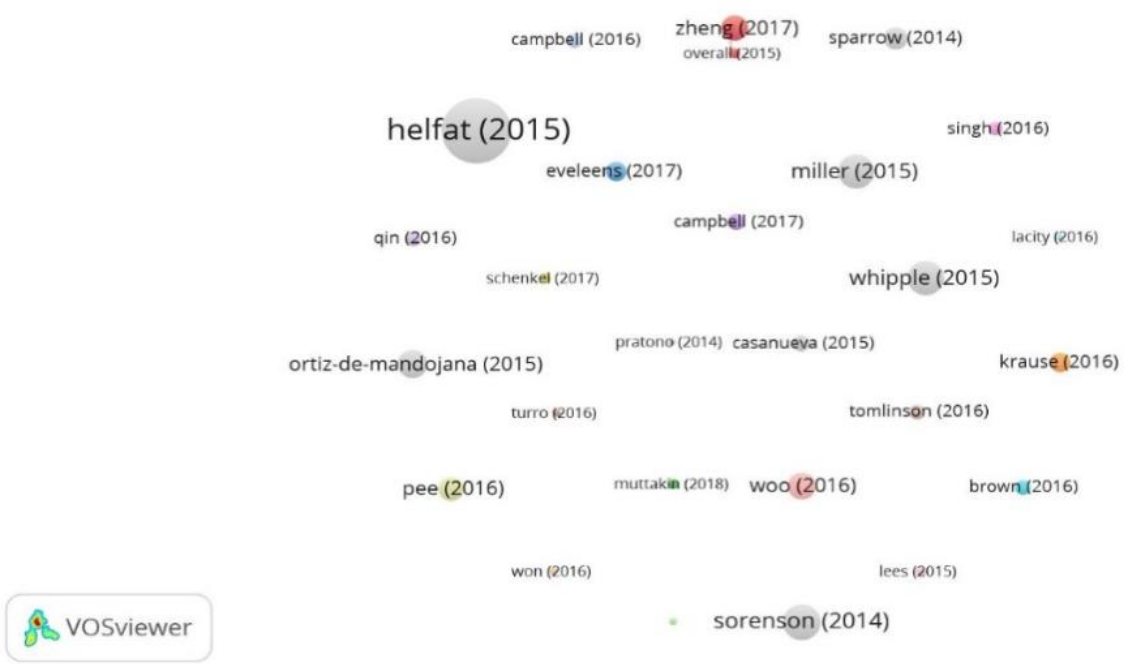

Fuente: (Base de datos Scopus) y VOSviewer

Una explicación de lo anterior puede ser el hecho de que el desempeño y las decisiones de los directivos influyen directamente en el éxito o fracaso de la organización, de ahí la relevancia para ellos de desarrollar y utilizar de manera eficiente su capital social como un recurso estratégico para instaurar relaciones sociales dentro y fuera de la empresa, las cuales pueden ser determinantes en la adquisición de conocimiento, la formación de alianzas y la transmisión de información. Basta recordar que los directivos juegan un papel clave como intermediarios de estas relaciones entre empleados, proveedores, clientes y demás actores involucrados.

De manera semejante, aunque en diferente grado, se considera que las relaciones sociales entre compradores, vendedores, empleados, tienen un impacto significativo en la innovación y el rendimiento de la empresa (Whipple et al, 2015; Overall, 2015; Tomlinson y Fai, 2016), tal y como se fundamenta en la revisión teórica. Asimismo, que para construir y mantener relaciones sociales sólidas y de calidad, son necesarias la comunicación, la confianza y el compromiso (Lees y Nuthall, 2015; Overall, 2015; Muldoon y Bauman, 2018). Es por esta razón algunas empresas están invirtiendo en el desarrollo de habilidades de gestión de relaciones internas entre sus empleados y directivos (Whipple et al, 2015), con el propósito de habilitarlos para un mejor desenvolvimiento en este ámbito.

Habría que resaltar la relevancia que tienen los recursos a nivel individual (Brieger y De Clercq, 2018), para ser más específicos la experiencia, habilidades y conocimiento que posee el personal de la empresa (Miller et al, 2015; Krause et al, 2016; Purg et al, 2016), como una capacidad altamente valorada por su aporte a la generación y sostenimiento del capital social, el cual como ya se ha mencionado, incide en la competitividad y 
rentabilidad de las empresas y, por ende, en su crecimiento y desarrollo.

Por lo que se refiere a las capacidades dinámicas, el análisis bibliométrico realizado arrojó que el capital social juega un papel preponderante en la creación de recursos estratégicos que contribuyen al proceso de innovación (Tomlinson y Fai, 2016; Zheng, Wu y Xie, 2017; Wang et al, 2018; Zhang y Guan, 2018) y gestión del conocimiento (Pee y Kankanhalli, 2016; Overall, 2015), ambas consideradas como capacidades dinámicas (Garzón, 2015), las cuales presentan un fuerte efecto en la creación de ventajas competitivas sostenibles.

Finalmente, otros autores como Helfat y Martin (2015), Turro, Alvarez y Urbano (2016), Singh y Rao (2016) y Schenkel y Teigland (2017) retoman el tema de las capacidades dinámicas de la empresa y su relación con el capital intelectual, humano y social, para posteriormente analizar su impacto en el desempeño tanto individual como organizacional. Siendo este último, una de las preocupaciones más recurrentes como se puede observar en los trabajos de Pratono y Mahmood (2014), Brown, Andersson y Jo (2016), Yasin et al (2016), Won (2016), Campbell y Park (2016), Jalali et al, (2017), Mahmood et al (2017), Abolghasemi et al (2018). En sus estudios, estos autores aluden al capital social como el conjunto de relaciones sociales tanto internas como externas que desarrolladas y cimentadas eficientemente, son fundamentales para el éxito de la organización.

\section{CONCLUSIONES}

De acuerdo con la revisión de la literatura realizada, se puede decir que el capital social es visto como un recurso firme y único como señala la TRC (Penrose, 1959; Andrews, 1971; Barney, 1991), es decir, como un recurso que puede ser utilizado de distintas maneras, cantidades y combinaciones para producir bienes y servicios dirigidos a un determinado mercado. El capital social cumple esta función al ser utilizado como un intangible que provee a la empresa de relaciones sociales, información y conocimiento para crear ventajas competitivas.

Asimismo, la posesión de ciertos recursos diferenciados como el caso de las relaciones sociales, no es una condición suficiente, aunque sí necesaria, para desarrollar una ventaja competitiva, pues estos requieren la existencia de capacidades que permitan primero identificar sus potencialidades, y luego, poder explotarlas al máximo. En esta revisión resultó común encontrar que estas capacidades se asocian con el enfoque de capacidades dinámicas, en el sentido de que algunas de ellas se consideran especiales o estratégicas, tal es el caso de la capacidad de innovación, la capacidad de aprendizaje o la gestión del conocimiento.

Respecto a la metodología utilizada, los resultados confirman que el análisis bibliométrico es una poderosa herramienta ya que facilita la recolección, selección y análisis de documentos científicos, para posteriormente elaborar planteamientos que vienen a enriquecer áreas poco estudiadas como la abordada en este estudio.

Por lo que a partir de dichos resultados, se contribuye a la generación de nuevo conocimiento en relación con una perspectiva más reciente del capital social por medio de la TRC, la cual pone de manifiesto que el capital social sigue viéndose como un conjunto de recursos y capacidades internas de la empresa pero que al mismo tiempo no 


\section{López-Inda, Karina Azucena, Alvarado Altamirano, Sergio, Fong Reinoso, Carlos y González \\ Álvarez, Eleazar}

puede darse de manera aislada y desconectada de una realidad en constante cambio $y$ evolución, sino que se hace necesario complementar su tratamiento con el enfoque de capacidades dinámicas el cual aporta, por un lado, una perspectiva empresarial $\mathrm{y}$, por otro, una visión más objetiva.

Este artículo también expone que las futuras investigaciones deben prestar mayor atención a la calidad y tipo de relaciones sociales que se deben cultivar y cómo es que se originan, en la idea de convertirlas en capital social útil. Asimismo, deben hacer hincapié en que no basta con acumular este capital, sino que se deben desarrollar capacidades únicas y diferenciadas para gestionarlo adecuadamente y construir con ello ventajas competitivas sostenibles.

\section{REFERENCIAS}

Abili, K. (2011). Social capital management in Iranian knowledge-based organizations' the electronic, Journal of Knowledge Management, 9(3), 203-210.

Abolghasemi M., Ismail S., Sharif, N., Kookhdan A. y Mardani A. (2018). Enhancing the performance of residential construction project through stakeholder satisfaction: The application of Structural Equation Modelling (SEM), Transformations in Business and Economics, 17(2), 107136.

Anand, V., Glick, W. y Manz, C. (2002). Thriving on the knowledge of outsiders: tapping organizational social capital. Academy of Management Executive, 16, 87-101.

Andrews, Kenneth R. (1971). El concepto de estrategia corporativa. Nueva York: Dow Jones-Irwin.

Arbeláez, M. y Onrubia, J. (2014). Análisis bibliométrico y de contenido. Dos metodologías complementarias para el análisis de la revista colombiana Educación y Cultura. Revista de Investigaciones UCM, 14(23), 14-31.

Barney, J. (1991). Firm resources and sustained competitive advantage.

Barney, J., Wright, M. y Ketchen, D. (2011). The future of resource-based theory: Revitalization or decline? Journal of Management, 77(5), 1299-1315.

Becattini, G. (2006). Vicisitudes y potencialidades de un concepto: el distrito industrial, Revista Economía Industrial, № 359, pp. 21-27.
Blanco-Mesa, F., Gil-Lafuente, A. y Merigó, J. (2017). Fuzzy decision making: A bibliometric-based review, Journal of Intelligent and Fuzzy Systems, 32(3), 2033-2050.

Bourdieu, P. (1980). Le capital social. In: Actes de la recherche en sciences sociales. Vol. 31, pp. 2-3. $<$ www.persee.fr/doc/arss_03355322_1980_num_31_1_2069>

Brieger, S. y De Clercq, D. (2018). Entrepreneurs' individuallevel resources and social value creation goals: The moderating role of cultural context, International Journal of Entrepreneurial Behaviour and Research, 25(2), 193216.

Brown W., Andersson F. y Jo S. (2016). Dimensions of Capacity in Nonprofit Human Service Organizations Voluntas, 27(6), 2889-2912.

Burvill, M., Jones-Evans, D. y Rowlands, H. (2018). Reconceptualising the principles of Penrose's (1959) theory and the resource-based view of the firm: The generation of a new conceptual framework, Journal of Small Business and Enterprise Development, 25 (6), 930959.

Campbell, J. y Park, J. (2016). Internal and external resources of competitive advantage for small business success: Validation across family ownership, International Journal of Entrepreneurship and Small Business, 27(4), 505-524.

Casanueva, C., Gallego, A. y Revilla, M. (2015). Access and mobilization of network resources and competitive advantage in hotels: A conceptual framework, International Journal of Contemporary Hospitality Management, 27(6), 1279-1300.

Castanias, R. y Helfat, C. (2001). The managerial rents model: theory and empirical analysis. Journal of Management 27(6), 661-678.

Coleman, J. (1988). Social capital in the creation of human capital. American Journal of Sociology, 94 (Supplement): S95-S120.

Florida, R. (2010). La clase creativa: la transformación de la cultura del trabajo y el ocio en el siglo XXI: Paidós.

Fong, C., Flores, K. y Cardoza, L. (2017). La teoría de recursos y capacidades: un análisis bibliométrico. Nova Scientia, 9(19), 411-440.

Garzón, M. (2015). Modelo de capacidades dinámicas. Revista Dimensión Empresarial, 13(1), 111-131.

Garrigos-Simon, F., Botella-Carrubi, M. y González-Cruz, T. (2018). Social capital, human capital, and sustainability: A bibliometric and visualization analysis, Sustainability, 10(12), 4751.

Grant, R. (1991). The Resource-Based Theory of Competitive Advantage: Implications for Strategy Formulation, California Management Review, 33(3), 114-135. 


\section{Capital social en la perspectiva de la teoría de recursos y capacidades: un análisis}

Granovetter, M. (1992). Problems of Explanation in Economic Sociology. En N. Nohria y R. Eccles [eds.]: Networks and Organizations: Structure, form and Action. Boston: Harvard Business School Press.

Habbershon, T. y Williams, M. (1999). A resource-based framework for assessing the strategic advantages of family firms, Family Business Review, 12(1), 1-25.

Helfat, C. y Peterat, M. (2003). The dynamic resource-based view: capability lifecycles, Strategic Management Journal, 24(10), 977-1010.

Helfat, C., Finkelstein, S., Mitchell, W., Peteraf, M., Singh, H., Teece, D., y Winter, S. (2007). Dynamic capabilities: Understanding strategic change in organizations. London: Blackwell.

Helfat C. y Martin J. (2015). Dynamic Managerial Capabilities: Review and Assessment of Managerial Impact on Strategic Change, Journal of Management, 41(5), 1281-1312.

Huy, Q. y Zott, C. (2019). Exploring the Affective Underpinnings of Dynamic Managerial Capabilities: How Managers' Emotion Regulation Behaviors Mobilize Resources for Their Firms, Strategic Management Journal, 40(10), 28-54.

Huynh T. y Patton D. (2015). Early-stage financing of university spin-offs: The impact of entrepreneurial capabilities and social networks of founding teams during start-ups, Proceedings of the European Conference on Innovation and Entrepreneurship, ECIE, 272-280.

Jalali A., Thurasamy R. y Jaafar M. (2017). The moderating effect of social capital in relation to entrepreneurial orientation and firm performance, Handbook of Research on Small and Medium Enterprises in Developing Countries, 82-116.

Krause R., Semadeni M. y Withers M. (2016). That special someone: When the board views its chair as a resource, Strategic Management Journal, 37(9), 1990-2002.

Lee, Choonwoo, Lee, Kyungmook y Pennings, J. (2001). Internal capabilities, external networks and performance: A study on technology-Based Ventures, Strategic Management Journal, 22(6-7), 615-640.

Lees, N. y Nuthall, P. (2015). Case study analysis on supplier commitment to added value agri-food supply chains in New Zealand, Agricultural and Food Economics, 3(4).

Mahmood, R., Mohd Zahari, A., Yaacob, N. y Mat Zin, S. (2017). Small firm performance: an empirical analysis in Malaysian housing construction industry, International Journal of Housing Markets and Analysis, 10(1), 50-65.

Mahoney, J. y Pandian, J. (1992). The resource-based view within the conversation of strategic management. Strategic Management Journal, 13(5), 363-380.
Merigó, J., Gil-Lafuente, A. y Yager, R. (2015). An overview of fuzzy research with bibliometric indicators, Applied Soft Computing, 27, 420-433.

Miller, D., Xu, X. y Mehrotra, V. (2015). When is human capital a valuable resource? the performance effects of Ivy league selection among celebrated CEOs, Strategic Management Journal, 36(6), 930-944.

Mintzberg, H., Lampel, J. y Ahlstrand, B. (1999). Safari a la estrategia. Buenos Aires, Argentina: Ediciones Granica.

Muldoon, J., Bauman, A. y Lucy, C. (2018). Entrepreneurial ecosystem: do you trust or distrust?, Journal of Enterprising Communities, 12(2), 158-177.

Muttakin, M., Khan, A. y Mihret, D. (2018). The Effect of Board Capital and CEO Power on Corporate Social Responsibility Disclosures, Journal of Business Ethics, 150(1), 41-56.

Nahapiet, J. y Ghoshal, S. (1998). Social capital, intellectual capital, and the organizational advantage. Academy Management Review. 23, 242-266.

Nelson, R. y Winter, S. (1982). An evolutionary theory of economic change. cambridge, Massachusetts: The Belknap Press of Harvard University Press.

Ostrom, E. y Ahn, T. (2003). Una perspectiva del capital social desde las ciencias sociales: capital social y acción colectiva. Revista Mexicana de Sociología, 65(1), 155-233.

Overall, J. (2015). A conceptual framework of innovation and performance: The importance of leadership, relationship quality, and knowledge management, Academy of Entrepreneurship Journal, 21(2), 41-54.

Pee, L. y Kankanhalli, A. (2016). Interactions among factors influencing knowledge management in public-sector organizations: A resource-based view, Government Information Quarterly, 33(1), 188-199.

Penrose, E. (1959). The theory of the growth of the firm. Oxford: Oxford University Press.

Peteraf, M. (1993). The cornerstones of competitive advantage: a resource-based view, Strategic Management Journal, 14(3), 179-191.

Peteraf, M., Di Stefano, G. y Verona, G. (2013). The elephant in the room of dynamic capabilities: bringing two diverging conversations together, Strategic Management Journal, 34(12), 1389-1410.

Pineda D. (2015). Análisis bibliométrico para la identificación de factores de innovación en la industria alimenticia, $A D$ minister, 27, 95-126.

Porter, M. (1990). The competitive advantage of nations. Harvard Business Review, marzo-abril. New York: Free Press.

Portugal, M., Ribeiro, F., Kramer, B., y Almeida, M. (2016). A bibliometric study of the resource-based view (RBV) in 


\section{López-Inda, Karina Azucena, Alvarado Altamirano, Sergio, Fong Reinoso, Carlos y González \\ Álvarez, Eleazar}

International Business Research using Barney (1991) as a key marker, Innovar, 26(61), 131-144

Pratono, A. y Mahmood, R. (2014). Social capital and firm performance: Moderating effect of environmental turbulence, Asian Social Science, 10(19), 59-68.

Purg, D., Saginova, O., Skorobogatykh, I. y Musatova, Z. (2016). Family owned hidden champions in Russia: Innovations, human capital and internationalization, Indian Journal of Science and Technology, 9(12).

Putnam, R. (1993). Making democracy work: Civic traditions in modern Italy. Princeton University Press. New Jersey.

Rumelt, R. (1984). Towards a strategic theory of the firm in Competitive Strategic Management, Lamb R. PrenticeHall.

Schenkel, A. y Teigland, R. (2017). Why doesn't downsizing deliver? A multi-level model integrating downsizing, social capital, dynamic capabilities, and firm performance, International Journal of Human Resource Management, 28(7), 1065-1107.

Singh, B. y Rao, M. (2016). Effect of intellectual capital on dynamic capabilities, Journal of Organizational Change Management, 29(2), 129-149.

Sorenson, O. (2014). Status and reputation: Synonyms or separate concepts? Strategic Organization, 12(1), 62-69.

Teece, D. y Pisano, G. (1994). The dynamic capabilities of firms: An introduction. Industrial and Corporate Change, 3, 537-556.

Teece, D., Pisano, G. y Shuen, A. (1997). Dynamic capabilities and strategic management. Strategic Management Journal, $18,509-533$.

Tomlinson, P. y Fai F. (2016). The impact of deep vertical supply chain relationships upon focal-firm innovation performance, $R$ and D Management, 46, 277-290.

Turro, A., Alvarez, C. y Urbano, D. (2016). Intrapreneurship in the Spanish context: a regional analysis, Entrepreneurship and Regional Development, 28, 380402.

van Nunen (2017). Bibliometric analysis of safety culture research, Safety Science, 108, 248-258

Ventura, J. (2008). Análisis estratégico de la empresa (1ª ed.). Madrid: Ediciones Paraninfo.

Wang, B. y Zhao, P. (2018). Worldwide research productivity in the field of back pain: A bibliometric analysis, Medicine, 97(40), e11566.

Wang, L., Huang, M. y Liu, M. (2018). How the founders' social capital affects the success of open-source projects: A resource-based view of project teams, Electronic Commerce Research and Applications, 28, 114-126.
Wernerfelt, B. (1984). A resource-based view of the firm. Strategic Management Journal, 5, 171-180.

Whipple, J., Wiedmer, R. y Boyer K. (2015). A Dyadic investigation of collaborative competence, social capital, and performance in buyer-supplier relationships, Journal of Supply Chain Management, 51(2), 3-21.

Won, D. (2016). An analysis on antecedents path of export performance and moderating effects of social capital in materials and components SMEs, Journal of Distribution Science, 14(2), 135-44.

Yasin, N., Quoquab, F., Kamarudin, S. y Mohammad, J. (2017). Overcoming the shortfall of resources for the growth of Malaysian university start-up companies, Advanced Science Letters, 23(4), 2995-2998.

Zhang, J. y Guan, J. (2018). The time-varying impacts of government incentives on innovation, Technological Forecasting and Social Change, 135, 132-144.

Zheng, J., Wu, G. y Xie, H. (2017). Impacts of leadership on project-based organizational innovation performance: The mediator of knowledge sharing and moderator of social capital, Sustainability (Switzerland), 9(10), 18-93.

Zollo, M., y Winter, S. (2002). Deliberate learning and the evolution of dynamic capabilities. Organization Science, $13,339-351$ 